\title{
Current status on the adoption of high energy devices in Italy: An Italian Society for Endoscopic Surgery and New Technologies (SICE) national survey
}

\author{
Emanuele Botteri ${ }^{1} \cdot$ Mauro Podda $^{2} \cdot$ Alberto Arezzo $^{3} \cdot$ Nereo Vettoretto $^{1} \cdot$ Alberto Sartori $^{4} \cdot$ Antonino Agrusa $^{5}$. \\ Marco Ettore Allaix ${ }^{3}$. Gabriele Anania ${ }^{6}$. Riccardo Brachet Contul ${ }^{7}$. Valerio Caracino ${ }^{8}$. Elisa Cassinotti ${ }^{9}$. \\ Diego Cuccurullo ${ }^{10}$. Giancarlo D'Ambrosio ${ }^{11}$. Marco Milone ${ }^{12} \cdot$ Irnerio Muttillo $^{13}$. Wanda Luisa Petz ${ }^{14}$. \\ Marcello Pisano ${ }^{15} \cdot$ Mario Guerrieri $^{16} \cdot$ Gianfranco Silecchia $^{17} \cdot$ Ferdinando Agresta $^{18}$
}

Received: 22 July 2020 / Accepted: 21 October 2020 / Published online: 5 November 2020

(c) Springer Science+Business Media, LLC, part of Springer Nature 2020

\begin{abstract}
Background In the past three decades, different High Energy Devices (HED) have been introduced in surgical practice to improve the efficiency of surgical procedures. HED allow vessel sealing, coagulation and transection as well as an efficient tissue dissection. This survey was designed to verify the current status on the adoption of HED in Italy.

Methods A survey was conducted across Italian general surgery units. The questionnaire was composed of three sections (general information, elective surgery, emergency surgery) including 44 questions. Only one member per each surgery unit was allowed to complete the questionnaire. For elective procedures, the survey included questions on thyroid surgery, lower and upper GI surgery, proctologic surgery, adrenal gland surgery, pancreatic and hepatobiliary surgery, cholecystectomy, abdominal wall surgery and breast surgery. Appendectomy, cholecystectomy for acute cholecystitis and bowel obstruction due to adhesions were considered for emergency surgery. The list of alternatives for every single question included a percentage category as follows: " $<25 \%, 25-50 \%, 51-75 \%$ or $>75 \%$ ", both for open and minimally-invasive surgery.

Results A total of 113 surgical units completed the questionnaire. The reported use of HED was high both in open and minimally-invasive upper and lower GI surgery. Similarly, HED were widely used in minimally-invasive pancreatic and adrenal surgery. The use of HED was wider in minimally-invasive hepatic and biliary tree surgery compared to open surgery, whereas the majority of the respondents reported the use of any type of HED in less than $25 \%$ of elective cholecystectomies. HED were only rarely employed also in the majority of emergency open and laparoscopic procedures, including cholecystectomy, appendectomy, and adhesiolysis. Similarly, very few respondents declared to use HED in abdominal wall surgery and proctology. The distribution of the most used type of HED varied among the different surgical interventions. US HED were mostly used in thyroid, upper GI, and adrenal surgery. A relevant use of H-US/RF devices was reported in lower GI, pancreatic, hepatobiliary and breast surgery. RF HED were the preferred choice in proctology.

Conclusion HED are extensively used in minimally-invasive elective surgery involving the upper and lower GI tract, liver, pancreas and adrenal gland. Nowadays, reasons for choosing a specific HED in clinical practice rely on several aspects, including surgeon's preference, economic features, and specific drawbacks of the energy employed.
\end{abstract}

Keywords High energy devices · Electrosurgery $\cdot$ Bipolar vessels sealing systems $\cdot$ Harmonic scalpel $\cdot$ Radiofrequency electrosurgery $\cdot$ Survey

All authors are members or past members of the Executive Board of the Società Italiana chirurgia Endoscopica e Nuove Tecnologie (SICE).

Emanuele Botteri

e.botteri@libero.it

Extended author information available on the last page of the article
In the past three decades, different High Energy Devices (HED) have been introduced in surgical practice to improve the efficiency of surgical procedures. HED allow vessel sealing, coagulation, and transection as well as an efficient tissue dissection. They can reduce operative time and postoperative complications [1-3]. 
Three distinct technologies are mainly involved in building HED: ultrasonic (US), radiofrequency (RF), and hybrid US/RF energy (H-US/RF).

US devices work based on the transformation of electrical energy into high-frequency $(55000 \mathrm{kHz})$ frictional energy. The vibrating blades allow the denaturation of hydrogen bonds in tissue and blood vessel proteins with the result that the coagulum seals the lumen of vessels up to $5 \mathrm{~mm}$ in diameter [4]. RF instruments apply bipolar high electric current $(4 \mathrm{~A})$ at a low voltage $(<200 \mathrm{~V})$. This way, energy denatures the collagen and elastin within the blood vessel wall and can seal vessels with a diameter of up to $7 \mathrm{~mm}[5,6]$.

Nowadays, a device that integrates both US and advanced bipolar energy in a unique instrument (H-US/ RF) is also available: it allows to cut tissue with US energy on the one hand and seal vessels with bipolar energy.

There are several disadvantages of HED instruments for hemostasis, including the relatively high costs due to the non-reusability of disposable instruments, and the generation of smoke, which may compromise visibility [7].

Today the choice among the HED is mainly based on the surgeon's preference. In fact, only a few studies that compare the different technologies have been published to date [8-10] without finding a clear advantage for the use of US or RF. There is also a lack of multidimensional evaluation of available instruments. The origins of the present work rely on the assumption that nowadays none knows how many surgeons choose HED in their clinically practice. Moreover, the use of HED in specific field, such as urgency, is not reported in high-quality literature.

The present study aims to report and critically appraise the results of a web-survey promoted among Italian surgeons, endorsed by the Italian Society for Endoscopic Surgery and New Technologies (Società Italiana di Chirurgia Endoscopica e Nuove Tecnologie, SICE), about the current habits of Italian surgeons in the use of HED. This work represents the scaffold for an HTA or another multidimensional evaluation for HED.

\section{Material and methods}

The Executive Board of SICE promoted a web-based survey to investigate how surgeons working in general surgery units across Italy currently use HED in daily clinical practice. Their participation remained voluntary, as no incentives were offered to participants. All parts of the study, and the present manuscript have been checked and presented according to the E-Surveys Checklist for Reporting Results of Internet (CHERRIES) [11].

\section{Questionnaire development}

The study steering committee (EB, NV, FA, MP, AR, AS) developed the questionnaire using remote brainstorming, after identifying the questions to include. The technical functionality of the electronic questionnaire was tested before the invitations were sent. Once an agreement was reached, the questionnaire was completed using Google Form (Google LLC, Mountain View, California US).

The questionnaire included 44 questions divided into three sections (general information, elective surgery, emergency surgery) (Table 1). Only closed-ended questions were used. The list of alternatives for every single quantitative question included a percentage category as follows: " $<25 \%, 25-50 \%, 51-75 \%$ or $>75 \%$ ", both for open and minimally-invasive surgery. The steering committee decided to use ranges of predetermined percentages to allow a more accessible aggregation of the information collected. Each field required to specify the most used HED type choosing between US, RF, or H-US/RF.

If one kind of surgery was not performed at the surgical unit, this would be classified as NA (Not Applicable).

Among elective procedures, the survey included questions on thyroid surgery, lower and upper GI surgery, proctologic surgery, adrenal gland surgery, pancreatic and hepatobiliary surgery, abdominal wall surgery, and breast surgery. Elective cholecystectomy was listed separately from the hepatobiliary section.

According to the distribution of emergency surgical operations, appendectomy, cholecystectomy for acute cholecystitis, and bowel obstruction due to adhesions were considered representative for emergency surgery. The estimated time to complete the survey was 7-9 $\mathrm{min}$.

\section{Study circulation and data handling}

On February 24th, 2020, the questionnaire was available online and open for completion until April 14th, 2020. The link (https://docs.google.com/forms/d/13TBiow0AC Al-ah47cAgsgXat7KpQyyNarIISSKeG_nI/edit) was circulated through personal email invitations to the members of the SICE across the country by the SICE secretary, including four reminders sent during the opening of the questionnaire. The link to complete the questionnaire was also always available in the area of the SICE website (https ://siceitalia.com), a website dedicated to the dissemination of updates on scientific research regarding minimallyinvasive surgery and surgical innovations, mainly visited by surgeons with a particular interest in laparoscopic and minimally-invasive techniques. The SICE regional coordinators were involved for a better spread of the survey. 
Table 1 List of questions proposed in the survey

1. Upper-GI surgery

1.1 How frequently are HEDs used in open Upper-GI surgery at your department?

$\begin{array}{lllll}<25 \% & 25-50 \% & 51-75 \% & >75 \% & \text { NA } \\ <25 \% & 25-50 \% & 51-75 \% & >75 \% & \text { NA } \\ \text { US } & \text { RF } & \text { H-US/RF } & & \end{array}$

1.3 With regard to the percentage of HEDs used in Upper-GI surgery, what type of energy is preferentially employed?

\section{Lower-GI surgery}

2.1 How frequently are HEDs used in open Lower-GI surgery at your department?

2.2 How frequently are HEDs used in minimally invasive Lower-GI surgery at your department?

2.3 With regard to the percentage of HEDs used in Lower-GI surgery, what type of energy is preferentially employed?

\section{Proctology}

3.1 How frequently are HEDs used in proctology at your department?

3.2 With regard to the percentage of HEDs used in proctology, what type of energy is preferentially employed?

4. Thyroid surgery

4.1 How frequently are HEDs used in open thyroid surgery at your department?

4.2 How frequently are HEDs used in minimally invasive thyroid surgery at your department?

4.3 With regard to the percentage of HEDs used in thyroid surgery, what type of energy is preferentially employed?

\section{Adrenal gland surgery}

5.1 How frequently are HEDs used in open adrenal gland surgery at your department?

5.2 How frequently are HEDs used in minimally invasive adrenal gland surgery at your department?

5.3 With regard to the percentage of HEDs used in adrenal gland surgery, what type of energy is preferentially employed?

6. Breast surgery

6.1 How frequently are HEDs used in breast surgery at your department?

6.2 With regard to the percentage of HEDs used in breast surgery, what type of energy is preferentially employed?

\section{Pancreatic surgery}

7.1 How frequently are HEDs used in open pancreatic surgery at your department?

7.2 How frequently are HEDs used in minimally invasive pancreatic surgery at your department?

7.3 With regard to the percentage of HEDs used in pancreatic surgery, what type of energy is preferentially employed?

\section{Hepatobiliary surgery}

8.1 How frequently are HEDs used in open hepatobiliary surgery at your department?

8.2 How frequently are HEDs used in minimally invasive hepatobiliary surgery at your department?

8.3 With regard to the percentage of HEDs used in hepatobiliary surgery, what type of energy is preferentially employed?

9. Cholecystectomy

9.1 How frequently are HEDs used in open cholecystectomy for cholelithiasis at your department?

9.2 How frequently are HEDs used in minimally invasive cholecystectomy for cholelithiasis at your department?

9.3 With regard to the percentage of HEDs used in cholecystectomy for cholelithiasis, what type of energy is preferentially employed?

10. Abdominal wall surgery

10.1.1 How frequently are HEDs used in open inguinal hernia repair at your department?

10.1.2 How frequently are HEDs used in minimally invasive inguinal hernia repair at your department?

10.1.3 With regard to the percentage of HEDs used in inguinal hernia repair, what type of energy is preferentially employed?

10.2.1 How frequently are HEDs used in open incisional hernia repair at your department?

$\begin{array}{lllll}<25 \% & 25-50 \% & 51-75 \% & >75 \% & \text { NA } \\ <25 \% & 25-50 \% & 51-75 \% & >75 \% & \text { NA } \\ \text { US } & \text { RF } & \text { H-US/RF } & & \end{array}$

$<25 \% \quad 25-50 \% \quad 51-75 \% \quad>75 \% \quad \mathrm{NA}$

US RF H-US/RF

$\begin{array}{lllll}<25 \% & 25-50 \% & 51-75 \% & >75 \% & \text { NA } \\ <25 \% & 25-50 \% & 51-75 \% & >75 \% & \text { NA } \\ \text { US } & \text { RF } & \text { H-US/RF } & & \end{array}$

$<25 \% \quad 25-50 \% \quad 51-75 \% \quad>75 \% \quad \mathrm{NA}$

$<25 \% \quad 25-50 \% \quad 51-75 \% \quad>75 \% \quad$ NA

US RF H-US/RF

$<25 \% \quad 25-50 \% \quad 51-75 \% \quad>75 \% \quad$ NA

US RF H-US/RF

$<25 \% \quad 25-50 \% \quad 51-75 \% \quad>75 \% \quad$ NA

$\begin{array}{llll}<25 \% & 25-50 \% & 51-75 \% \quad>75 \% & \mathrm{NA}\end{array}$

US RF H-US/RF

$<25 \% \quad 25-50 \% \quad 51-75 \% \quad>75 \% \quad$ NA

$<25 \% \quad 25-50 \% \quad 51-75 \% \quad>75 \%$ NA

US RF H-US/RF

$<25 \% \quad 25-50 \% \quad 51-75 \% \quad>75 \% \quad$ NA

$<25 \% \quad 25-50 \% \quad 51-75 \% \quad>75 \% \quad$ NA

US RF H-US/RF

$<25 \% \quad 25-50 \% \quad 51-75 \% \quad>75 \%$ NA

$<25 \% \quad 25-50 \% \quad 51-75 \% \quad>75 \% \quad$ NA

US RF H-US/RF

$<25 \% \quad 25-50 \% \quad 51-75 \% \quad>75 \% \quad$ NA 
Table 1 (continued)

10.2.2 How frequently are HEDs used in minimally invasive incisional hernia repair at your depart- $\quad<25 \% \quad 25-50 \% \quad 51-75 \% \quad>75 \% \quad$ NA ment? 10.2.3 With regard to the percentage of HEDs used in incisional hernia repair, what type of energy $\quad$ US $\quad$ RF $\quad$ H-US/RF
is preferentially employed?

11. Emergency surgery

11.1.1 How frequently are HEDs used in open appendectomy at your department?

$<25 \% \quad 25-50 \% \quad 51-75 \% \quad>75 \% \quad \mathrm{NA}$

11.1.2 How frequently are HEDs used in minimally invasive appendectomy at your department?

11.1.3 With regard to the percentage of HEDs used in appendectomy, what type of energy is preferentially employed?

11.2.1 How frequently are HEDs used in open cholecystectomy for acute cholecystitis at your department?

11.2.2 How frequently are HEDs used in minimally invasive cholecystectomy for acute cholecystitis at your department?

11.2.3 With regard to the percentage of HEDs used in cholecystectomy for acute cholecystitis, what type of energy is preferentially employed?

11.3.1 How frequently are HEDs used in open adhesiolysis for small bowel obstruction at your department?

11.3.2 How frequently are HEDs used in minimally invasive adhesiolysis for small bowel obstruction at your department?

11.3.3 With regard to the percentage of HEDs used in adhesiolysis for small bowel obstruction, what type of energy is preferentially employed?

$<25 \% \quad 25-50 \% \quad 51-75 \% \quad>75 \% \quad \mathrm{NA}$

US RF H-US/RF

$<25 \% \quad 25-50 \% \quad 51-75 \% \quad>75 \% \quad$ NA

$<25 \% \quad 25-50 \% \quad 51-75 \% \quad>75 \% \quad$ NA

US RF H-US/RF

$<25 \% \quad 25-50 \% \quad 51-75 \% \quad>75 \% \quad$ NA

$<25 \% \quad 25-50 \% \quad 51-75 \% \quad>75 \% \quad$ NA

US RF H-US/RF
They were stimulated to give notice of the initiative by means of several social networks (Facebook, LinkedIn, Twitter) available. Only one member per each surgery unit was allowed to complete the questionnaire, as the aim of the study was to define the trend in the use of HED within the Italian departments of surgery, rather than the attitude of the single surgeon. Respondents remained anonymous. The invitation to participate came with a letter in which the types of surgical procedure for each question were listed. Moreover, we explained that the answers should not represent the preferences of the respondent, but rather should reflect the habits of the unit. In case of two answers coming from the same division the former would be erased.

A member of the steering committee (MP) downloaded the results of the survey and shared them with the other members of the steering committee for analysis of data and discussion.

\section{Results}

Results were reported using percentages and presented as histograms. In total, surgeons from 113 different surgical units completed the questionnaire. In Italy, there are 445 general surgery units. Assuming the survey news had reached all surgical units, we reported a $25 \%$ of reply to the survey. We received at least one answer from each Italian region.

\section{Upper GI surgery}

One hundred thirteen answers were registered (Fig. 1). Both in open and minimally-invasive upper GI surgery, the reported use of HED was extensive. In open surgery, 76.6\% of surgeons declared to use HED in more than $50 \%$ of procedures $(60.4 \%$ ' $>75 \%$ ', 16.2\% '51-75\%'). In minimallyinvasive surgery, $82.3 \%$ of participants declared to use HED in more than $75 \%$ of procedures. There was no real preference for the type of HED used, with a slight prevalence of US devices (38.9\% US and 35.4\% H-US/RF).

\section{Lower GI surgery}

One hundred thirteen answers were registered (Fig. 1). In open lower GI surgery, $77.9 \%$ of respondent surgeons stated to use HED in more than $50 \%$ of the procedures $(58.4 \%$ '>75\%', 19.5\% '51-75\%'). Results regarding minimallyinvasive surgery showed $89.4 \%$ of participants who declared to use HED in more than $75 \%$ of the procedures. There was no real preference for the type of HED used, $23.4 \% \mathrm{RF}$, $35.1 \% \mathrm{US}$, and $41.4 \% \mathrm{H}-\mathrm{US} / \mathrm{RF})$.

\section{Proctology}

One hundred twelve answers were registered (Fig. 1). 32.1\% of the centers declared to use HED in less than $25 \%$ of the procedures. The other frequencies were less represented in 


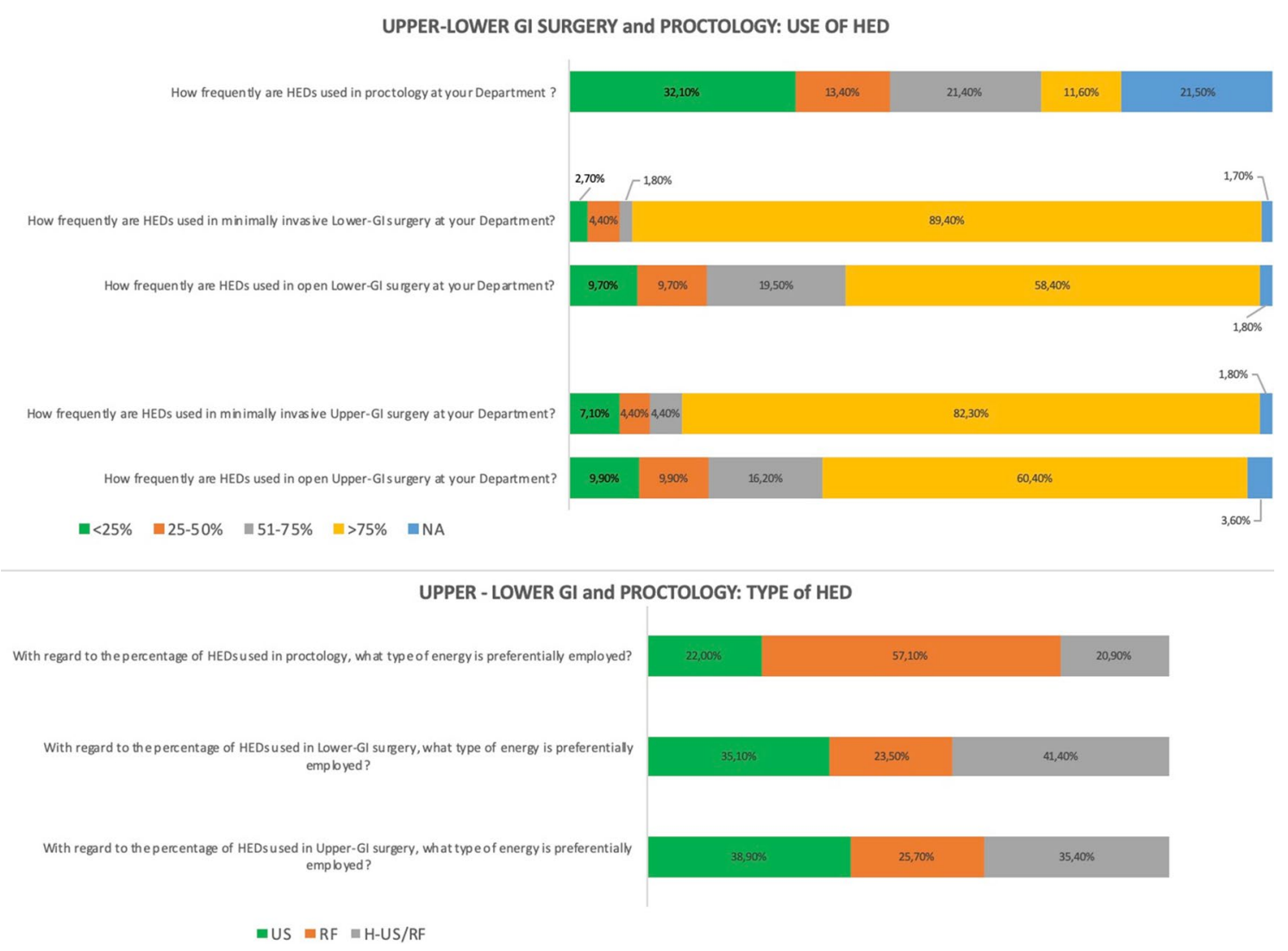

Fig. 1 Survey results for Upper GI Lower GI and Proctology

the aggregate results, with $13.4 \%$ for ' $25-50 \%$ ', $21.4 \%$ for ' $51-75 \%$ ', and $11.6 \%$ for ' $>75 \%$ '. When used, RF devices $(57.1 \%)$ were preferred.

\section{Pancreatic surgery}

One hundred twelve answers were registered (Fig. 2). As pancreatic surgery is a hyper-specialistic branch of general surgery, $25.9 \%$ of NA responses for open surgery, and $33.6 \%$ of NA for minimally-invasive surgery were registered. HED were mostly used in minimally-invasive pancreatic surgery, reaching $57.3 \%$ of ' $>75 \%$ ' answers. $37.6 \%$ of respondents who declared to perform pancreatic surgery used H-US/ RF HED, $36.6 \%$ stated to use US HED, and $25.8 \%$ of the respondents declared to use RF HED.

\section{Hepatic and biliary tree surgery}

One hundred thirteen answers were registered (Fig. 2). The stated use of HED was more extensive in minimally-invasive hepatic and biliary tree surgery $(59.8 \%)$ compared to open surgery (42.5\%). In open surgery, different levels of HED use were represented, with $15.9 \%, 13,3 \%$, and $12.4 \%$ respectively for ' $51-75 \%$ ', '25-50\%', '<25\%'. The use of US HED (41\%) and H-US/RF HED (43\%) were the most reported ones.

\section{Elective cholecystectomy}

One hundred thirteen answers were registered (Fig. 2). The reported use of HED was scarce in elective cholecystectomy. More than $70 \%$ of the respondents reported using any type of HED in less than $25 \%$ of the procedures, both for open and laparoscopic cholecystectomy.

\section{Abdominal wall surgery}

Both groin and incisional hernias were included in abdominal wall surgery in the present survey (Fig. 3). One hundred thirteen answers were registered. Decidedly few respondents declared to use HED in abdominal wall surgery $(<10 \%)$. A not negligible surgical unit rate does not perform abdominal wall surgery in our survey $(30.1 \%)$. 

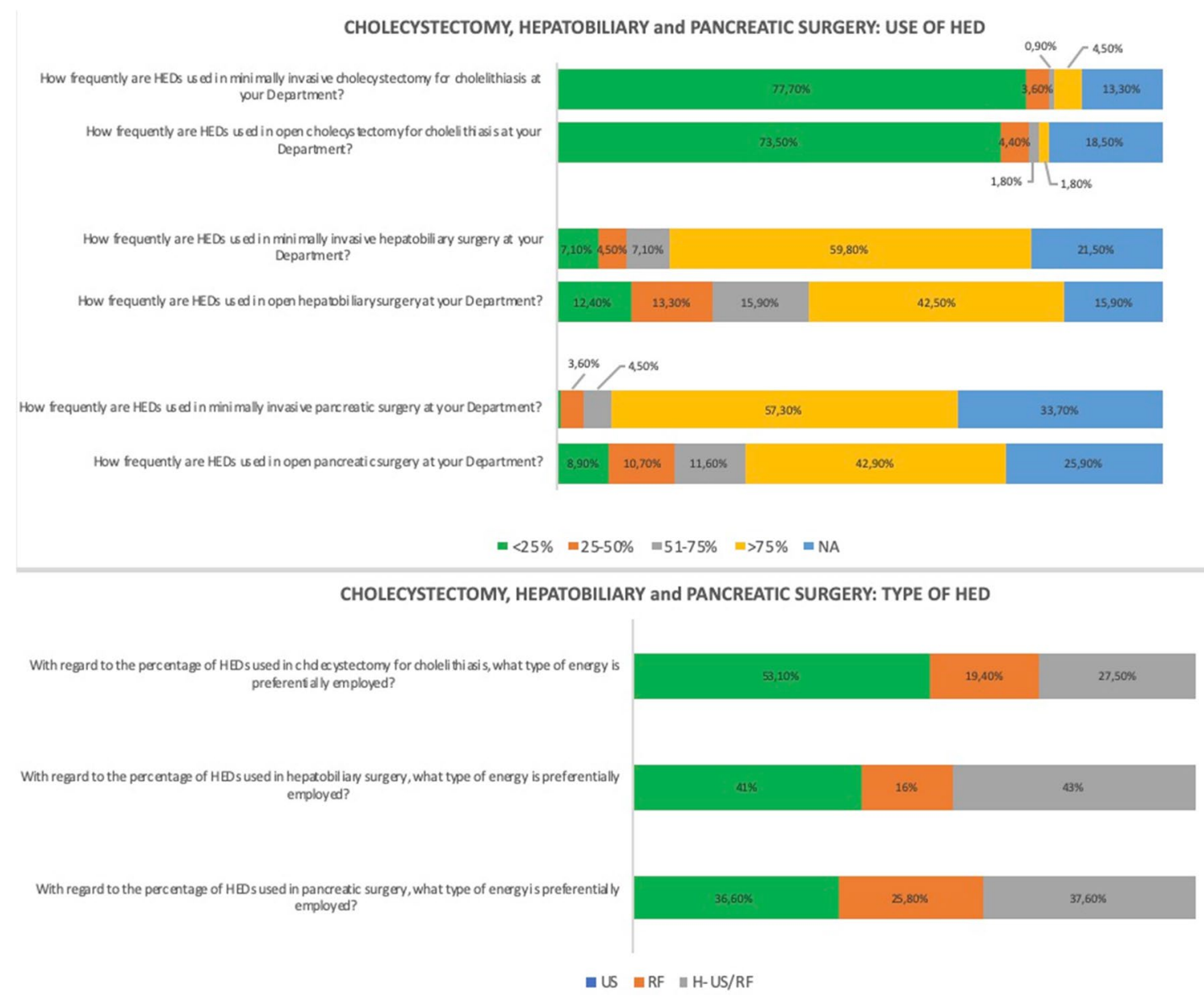

Fig. 2 Survey results for pancreatic, hepatobiliary surgery and elective cholecystectomies

\section{Thyroid surgery}

One hundred ten answers were registered (Fig. 4). 43.1\% of respondents declared to use HED for $>75 \%$ of open thyroidectomies.

Only a few centers declared to perform minimally-invasive thyroid surgery. $77.3 \%$ of NA answers were reported. Units that perform minimally-invasive thyroidectomy stated that US (50\%) was the most frequently used HED.

\section{Adrenal surgery}

One hundred eleven answers were registered (Fig. 4). Several surgical units do not perform adrenal surgery. NA was chosen in $37.8 \%$ and $28.6 \%$, respectively, for open and minimally-invasive surgery.

A broader tendency to use HED in minimally-invasive surgery $(61.6 \%$ of $>75 \%)$ than in open adrenal surgery (36.9\% of $>75 \%$ ) was reported. Significant use of US devices (41.8\% US and $29.7 \%$ H-US/RF) was noted in this field.

\section{Breast surgery}

One hundred eleven answers were registered (Fig. 4). The majority of our survey centers declared not to perform breast surgery (58.6\% of NA answers). The overall use of HED was low, as shown by the reported values of ' $<25 \%$ ' (23.4\%) and ' $25-50 \%$ ' (9.8\%).

\section{Appendectomy}

One hundred thirteen answers were registered (Fig. 5). HED are employed in $<25 \%$ of the open procedures according to $62.8 \%$ of the respondents, and in $<25 \%$ of the minimally-invasive procedures according to $56.3 \%$. A higher tendency in the use of HED in minimally-invasive than in open appendectomy was reported, as evidenced in the more significant percentage of the choices ' $51-75 \%$ ' and ' $>75 \%$ ' when compared to open surgery. US HED were the most frequently used (42\%), followed by RF (29\%) and H-US/RF. 
WALL SURGERY: USE OF HED

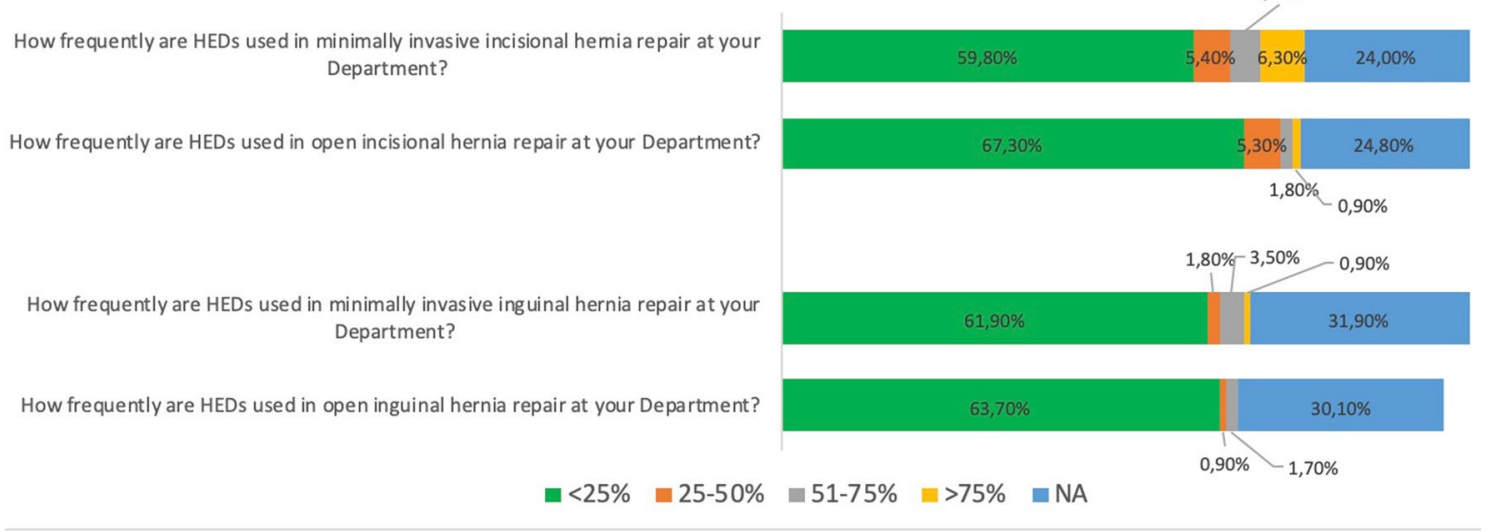

WALL SURGERY: TYPE OF HED

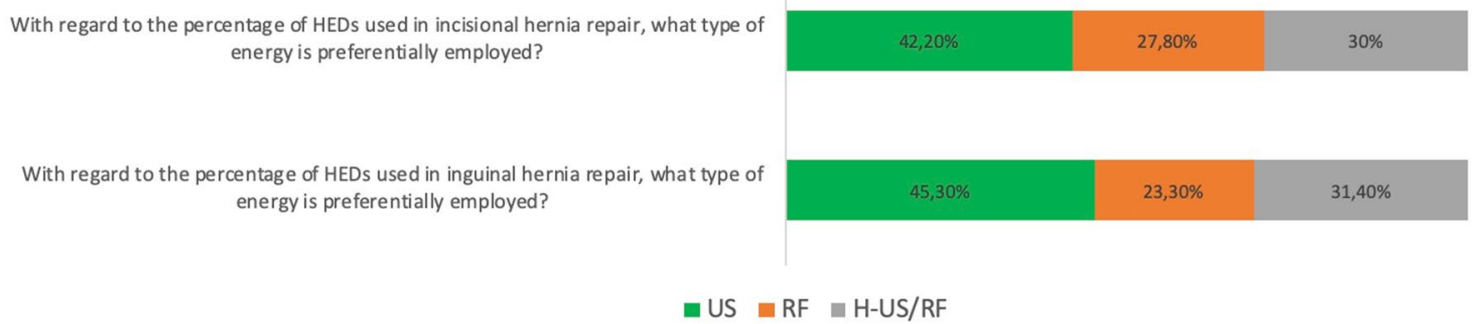

Fig. 3 Survey results for wall surgery

\section{BREAST, ADRENAL and THYROID SURGERY: USE OF HED}

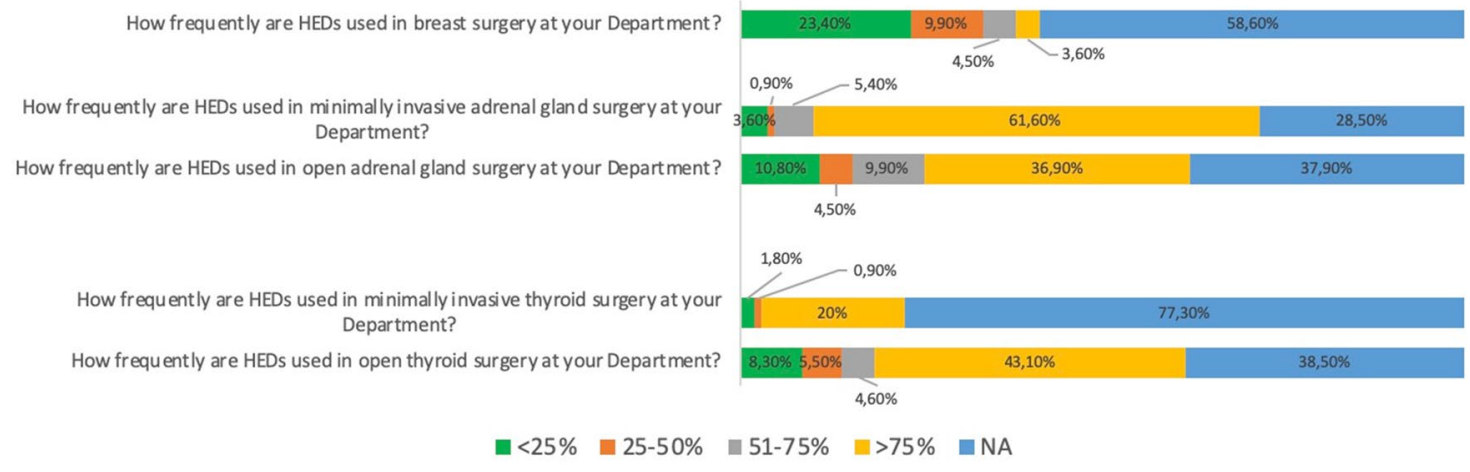

\section{BREAST, ADRENAL and THYROID SUREGRY: TYPE OF HED}

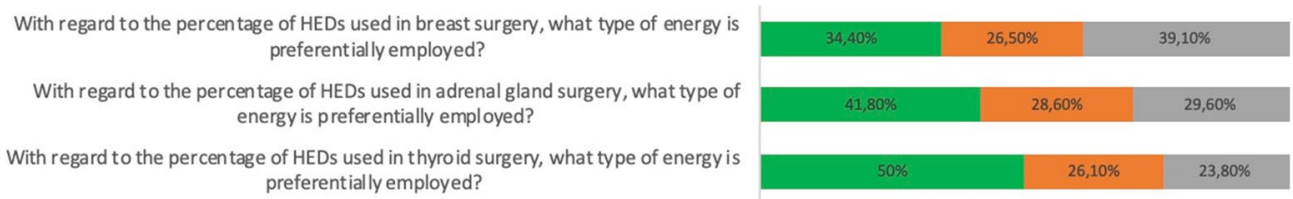

aU $\square$ RF $\square \mathrm{H}-\mathrm{US} / \mathrm{RF}$

Fig. 4 Survey results for breast, adrenal and thyroid surgery 
URGENT SURGERY: USE OF HED

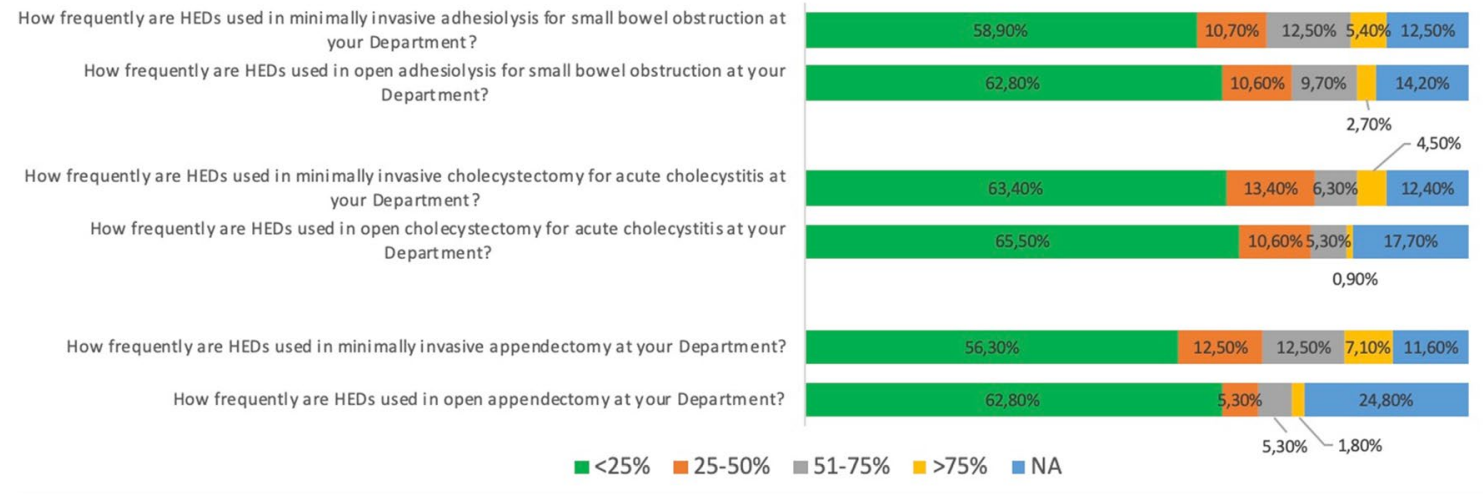

URGENT SURGERY: TYPE OF HED

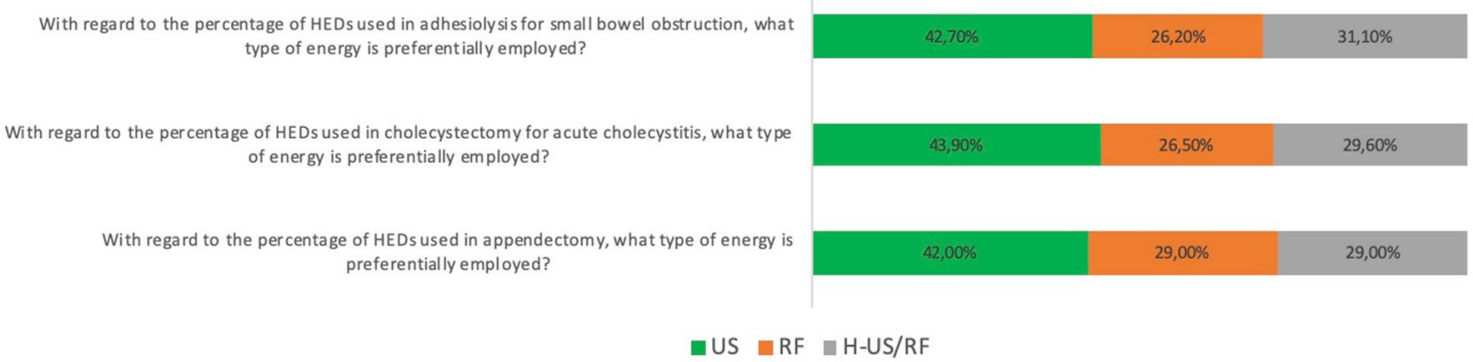

Fig. 5 Survey results for emergency surgery

\section{Cholecystectomy for acute cholecystitis}

One hundred thirteen answers were registered (Fig. 5). HED are only rarely employed $(<25 \%)$ in the majority of cholecystectomies, both in open and in minimally-invasive surgery ( $65.5 \%$ vs. $63.4 \%$ respectively). US HED are the most used (43.9\%), followed by H-US/RF (29.6\%) and RF $(26.5 \%)$.

\section{Adhesiolysis for small bowel obstruction}

One hundred thirteen answers were reported (Fig. 5). 62.8\% of the respondents declared to use HED in $<25 \%$ of the open adhesiolysis. The result is not far from that we reported for the minimally-invasive approach (58.9\%). HED were used in ' $>75 \%$ ' of minimally-invasive procedures according to $5.4 \%$ of respondents, whereas the rate drops to $2.7 \%$ for open surgery.

\section{Discussion}

We surveyed the Italian general surgery units to investigate the use of HED in daily clinical practice. The questions were divided into different type of surgery, and each of them required an independent answer based on a minimally-invasive approach or open surgery. One hundred thirteen surgery units joined the survey, representing about $25 \%$ of the Italian units.

The result falls in the usual rate of responses reported for email and web-surveys. Shih et al. showed that webbased surveys generally have an average response rate of $33 \%( \pm 22 \%)[12]$. We found that not all surgery units performed the same surgical procedure with either an open and minimally-invasive approach. So, for several pathologies, a high rate of NA answers was reported. There are different explanations for this phenomenon: for adrenal, pancreatic, breast, thyroid, and abdominal wall surgery, it could be due to the type of treatment performed only in hyper-specialistic centers or dedicated centers like breast unit. Conversely, for elective open cholecystectomy and open appendectomy, the reason lies in the minimally-invasive approach's better outcomes. Nowadays, laparoscopy is considered the gold standard in literature for both appendectomy and cholecystectomy $[13,14]$.

For upper and lower GI surgery HED are widely used, especially in laparoscopic surgery, reaching a rate of answer '> $75 \%$ ' more than $80 \%$. We observed a slight prevalence in $\mathrm{H}-\mathrm{RF} / \mathrm{US}$ use in lower GI surgery (41.4\%) and US devices in upper GI procedures (38.9\%).

Such a trend was not confirmed in proctology, where only $33 \%$ (11.6\% '> $75 \%$ ' and $21.4 \%$ ' $50-75 \%$ ') of the centers 
declared to use HED for more than $50 \%$ of surgical procedures. When HED were used in proctology, surgeons mainly choose RF devices (57.1\%).

As hemorrhoids mainly consist of vascular tissue, safe and quick surgery, avoiding the closure of hemorrhoidal vascular pedicles with stitches may be performed using the better sealing attitude of RF HED. Moreover, there are several surgical options available and nowadays, no single technique has been universally accepted as the best treatment. The therapeutic choice of treatment is largely dependent on the severity of the symptoms, the size of haemorrhoidal tissue, the extent of displacement and last but not least the surgeon's preference.

Reports from thyroid surgery showed that a not negligible rate of the involved centers declared to not perform thyroidectomy, primarily through a minimally-invasive approach (77.3\% NA). However, when minimally-invasive thyroidectomy is carried out, the use of HED is broad, and half of the surgeons choose US HED. US HED have a small and accurate tip that allows a careful dissection with a low lateral thermal spread to protect the laryngeal nerve.

HED were scarcely used in elective cholecystectomy and abdominal wall surgery, without differences between open and minimally-invasive approach. For cholecystectomy, the visceral dissection is carried out through a relatively low vascularized plane with only two anatomical structures to seal (cystic artery and cystic duct). Reason for which, cholecystectomy is safely performed with monopolar scalpel and clips without the need for HED in most cases.

The same considerations may be done for abdominal wall surgery: dissection is limited and performed safely following avascular planes with monopolar scissors or scalpel. When bleeding occurs, it can be controlled with bipolar claw.

The most frequent interventions for emergency surgery, such as appendectomy, adhesiolysis for acute small bowel obstruction, and cholecystectomy for cholecystitis, were considered. Even for these procedures, the use of HED was low both in open and laparoscopic approaches. Finally, adhesions are often managed with cold scissors to avoid thermal injuries to the adjacent organs. Thermal injuries could bring to late bowel perforation, which may require a reintervention.

According to Guidelines, no evidence-based recommendation to use HED routinely in these types of surgical operations can be formulated [13, 14].

It is still matter of debate whether HEDs represent an advantage or a risk for thermal injuries $[15,16]$. Also, the production of smoke is highly debated $[17,18]$ especially in time of COVID-19 pandemic [19-21]. Ultimately, the issue of high costs is still slackening the implementation of HED in surgical activity. Accurate evaluation for public health sustainability, defining cost-effectiveness for surgical devices in the hospital setting, is difficult and can be highly variable, while the possible reduction in operative time could be a minor advantage only.

Web-based surveys may be subject to relevant bias, especially from the non-representative nature of the web population and participants" self-selection (also called "the volunteer effect").

The study sample's representativeness is supported by the fact that at least one respondent from each Italian region was registered, making us confident that the respondents reflect the attitudes of the entire Italian surgical population.

Generally, web-based surveys are limited because of the possibility that the respondents (general surgeons performing mostly minimally-invasive surgery in this case) do not reflect, close enough, the target population (all general surgeons). In our case, the study sample was identified by the official list of SICE members, obtained from the society secretariat. In our survey, the questionnaire related to aspects concerning minimally-invasive surgery and open surgery, surgical emergencies, and proctology.

Although a high response rate minimizes the potential for bias and enhances the results of a web-based study, it has been remarked that there is no scientifically established minimum acceptable response rate, and it may not be associated with survey reliability or quality [21]. A further potential limitation of our study relies on the difficulty to accurately quantify the number of recipients, as we cannot argue the precise number of surgeons who read the invitation to complete the questionnaire. Although the questionnaire was open to all Italian surgical units, most of the responding surgeons were SICE members. Since the society's mission is to promote minimally-invasive surgery, this could represent a possible bias reflecting mainly the habit of laparoscopic surgeons in the use of HED. There is a lack of evidence-based data in the literature to support the use of RF, US o H-RF/US in different types of surgical operations, so nowadays, the choice of technology is based on the surgeon's preference. A complete cost-effective analysis is also lacking, and this could represent a motivation for future research.

\section{Conclusion}

The present study highlights the high use of HED in major elective minimally-invasive surgery. Choosing a specific HED in clinical practice relies on several aspects, including the surgeon's preference, economic features, and specific drawbacks of the energy employed.

Author contributions Steering Committee: AA AS EB MP FA NV, Analysis Data: EB MP, Manuscript Drafting: EB, MP, AR, AS, NV, FA. All the authors reviewed the manuscript for relevant intellectual contents, and approved the final version of the manuscript. 
Funding This research did not receive any specific grant from funding agencies in the public, commercial, or not-for-profit sectors.

\section{Compliance with ethical standards}

Disclosures Emanuele Botteri, Mauro Podda, Alberto Arezzo, Nereo Vettoretto, Alberto Sartori, Antonino Agrusa, Marco Ettore Allaix,Gabriele Anania, Riccardo Brachet Contul, Valerio Caracino, Elisa Cassinotti, Diego Cuccurullo, Giancarlo D'Ambrosio, Marco Milone, Irnerio Muttillo, Wanda Luisa Petz, Marcello Pisano, Mario Guerrieri, Gianfranco Silecchia and Ferdinando Agresta have no conflicts of interest or financial ties to disclose.

Ethics approval No ethical approval was required for this article.

Consent to participate The authors are accountable for all aspects of the work in ensuring that questions related to the accuracy or integrity of any part of the work are appropriately investigated and resolved.

\section{References}

1. Kennedy J, Buysse S, Lawes K et al (1999) Recent innovations in bipolar electrosurgery. Minim Invasive Ther Allied Technol 8:95-99

2. Carroll T, Ladner K, Meyers AD (2005) Alternative surgical dissection techniques. OtolaryngolClin N Am 38:397-411

3. Devassy R, Gopalakrishnan S, De Wilde RL (2015) Surgical efficacy among laparoscopic ultrasonic dissectors: are we advancing safely? A review of literature. J ObstetGynaecol India 65:293-300

4. Person B, Vivas DA, Ruiz D, Talcott M et al (2008) Comparison of four energy-based vascular sealing and cutting instruments: a porcine model. SurgEndosc 22:534-538

5. Harold KL, Pollinger H, Matthews BD et al (2003) Comparison of ultrasonic energy, bipolar thermal energy, and vascular clips for the hemostasis of small-, medium-, and large-sized arteries. SurgEndosc 17:1228-1230

6. Campbell PA, Cresswell AB, Frank TG et al (2003) Real time thermography during energized vessel sealing and dissection. SurgEndosc 17:1640-1645

7. Weld KJ, Dryer S, Ames CD et al (2007) Analysis of surgical smoke produced by various energy-based instruments and effect on laparoscopic visibility. J Endourol 21(3):347-351

8. Allaix ME, Edgar JB, Furneé E et al (2016) Energy sources for laparoscopic colorectal surgery: is one better than the others? J LaparoendAdvSurg 26:264-269
9. Di Lorenzo N, Franceschilli L, Allaix ME et al (2012) Radiofrequency versus ultrasonic energy in laparoscopic colorectal surgery: a meta-analysis of operative time and blood loss. SurgEndosc 26:2917-2924

10. Rimonda R, Arezzo A, Garrone C, et al. (2009) Electrothermal bipolar vessel sealing system vs. Harmonic scalpel in colorectal laparoscopic surgery: a prospective, randomized study. Dis Colon Rectum 52 (4): 657-661

11. Eysenbach G (2004) Improving the quality of web surveys: the checklist for reporting results of internet e-surveys (CHERRIES). J Med Internet Res 6(3):E34

12. Shih TH, Fan $X$ (2009) Comparing response rates in email and paper surveys: a meta-analysis. Educ Res Rev 4:26-40

13. Agresta F, Campanile FC, Vettoretto, et al (2015) Italian surgical society working group Laparoscopic cholecystectomy: consensus conference-based guidelines. Langebecks Arch Surg 400(4):429-453

14. Saverio Di, Podda M, De Simone B et al (2020) Diagnosis and treatment of acute appendicitis: 2020 updates of the WSES Jerusalem guidelines. World J Emergency Surg 15(1):27

15. Kim FJ, Sehrt D, da Silva RD et al (2015) Evaluation of emissivity and temperature profile of laparoscopic ultrasonic devices (blades and passive jaws). SurgEndosc 29(5):1179-1184

16. Tsirline VB, Lau KN, Swan RZ et al (2013) Evaluation of an innovative, cordless ultrasonic dissector. SurgInnov 20(5):524-529

17. Ott DE, Moss E, Martinez K (1998) Aerosol exposure from an ultrasonically activated (Harmonic) device. J Am AssocGynecolLaparosc 5(1):29-32

18. Kim F, David S, Pompeo A, Molina W (2012) Comparison of surgical plume among laparoscopic ultrasonic dissectors using a real-time digital quantitative technology. SurgEndosc 26(12):3408-3412

19. Mintz Y, Arezzo A, Boni L, Chand M, Brodie R, Fingerhut A, the Technology Committee of the European Association for Endoscopic Surgery (2020) A low-cost, safe, and effective method for smoke evacuation in laparoscopic surgery for suspected coronavirus patients. Ann Surg 272(1):e7-e8

20. Campanile FC, Podda M, Arezzo A et al (2020) Acute cholecystitis during COVID-19 pandemic: a multisocietary position statement. World J EmergSurg 15(1):38

21. Botteri E, Podda M, Sartori A (2020) The COVID-19 pandemic should not take us back to prelaparoscopic era. J Trauma Acute Care Surg 89(2):273-274.

Publisher's Note Springer Nature remains neutral with regard to jurisdictional claims in published maps and institutional affiliations.

\section{Affiliations}

Emanuele Botteri ${ }^{1} \cdot$ Mauro Podda $^{2} \cdot$ Alberto Arezzo $^{3} \cdot$ Nereo Vettoretto $^{1} \cdot$ Alberto Sartori $^{4} \cdot$ Antonino Agrusa $^{5}$. Marco Ettore Allaix ${ }^{3}$. Gabriele Anania ${ }^{6} \cdot$ Riccardo Brachet Contul $^{7}$. Valerio Caracino ${ }^{8}$. Elisa Cassinotti ${ }^{9}$. Diego Cuccurullo ${ }^{10}$. Giancarlo D'Ambrosio ${ }^{11}$. Marco Milone ${ }^{12}$. Irnerio Muttillo ${ }^{13}$. Wanda Luisa Petz ${ }^{14}$. Marcello Pisano ${ }^{15} \cdot$ Mario Guerrieri $^{16} \cdot$ Gianfranco Silecchia $^{17} \cdot$ Ferdinando Agresta $^{18}$

1 General Surgery, ASST Spedali Civili Di Brescia, Montichiari, Italy

2 Department of Emergency Surgery, Azienda Ospedaliero-Universitaria Di Cagliari, Policlinico Universitario Di Monserrato "Duilio Casula" University of Cagliari, Cagliari, Italy
3 Department of Surgical Sciences, University of Torino, Turin, Italy

4 Department of General Surgery, Ospedale Di Montebelluna, Montebelluna, Italy 
5 Department of General and Emergency Surgery, University of Palermo, Palermo, Italy

6 Department of Morphology, Surgery and Experimental Medicine, University of Ferrara, Ferrara, Italy

7 Department of General and Emergency Surgery, Ospedale Regionale Umberto Parini, Aosta, Italy

$8 \quad 2^{\circ}$ General Surgery, Hospital "Spirito Santo", Pescara, Italy

9 Chirurgia Generale, Fondazione IRCCS Ca' Granda Ospedale Maggiore Policlinico, Milan, Italy

10 Department of General Surgery, Ospedali Dei Colli Monaldi Hospital, Naples, Italy

11 Department of General Surgery, Surgical Specialties and Organ Transplantation, Rome, Italy

12 Department of Clinical Medicine and Surgery, Federico II University of Naples, Naples, Italy
13 Department of General and Emergency Surgery, Ospedale San Filippo Neri, Roma, Italy

14 Department of Surgery, IEO, European Institute of Oncology IRCCS, Milan, Italy

15 Department of General Surgery, Ospedale San Marcellino di Muravera, Cagliari, Italy

16 Department of General Surgery, Università Politecnica delle Marche, Ancona, Italy

17 Department of Medico-Surgical Sciences and Biotechnologies, University La Sapienza of Rome, Latina, Italy

18 Department of General Surgery, Ospedale Civile, Adria, Italy 